Sigma Mesa: Background Elemental

Concentrations in Soil and

Vegetation, 1979

Roger W. Ferenbaugh

Ernest S. Gladney

George H. Brooks, Jr. 


\section{DISCLAIMER}

This report was prepared as an account of work sponsored by an agency of the United States Government. Neither the United States Government nor any agency Thereof, nor any of their employees, makes any warranty, express or implied, or assumes any legal liability or responsibility for the accuracy, completeness, or usefulness of any information, apparatus, product, or process disclosed, or represents that its use would not infringe privately owned rights. Reference herein to any specific commercial product, process, or service by trade name, trademark, manufacturer, or otherwise does not necessarily constitute or imply its endorsement, recommendation, or favoring by the United States Government or any agency thereof. The views and opinions of authors expressed herein do not necessarily state or reflect those of the United States Government or any agency thereof. 


\section{DISCLAIMER}

Portions of this document may be illegible in electronic image products. Images are produced from the best available original document. 


\title{
SIGMA MESA: \\ BACKGROUND ELEMENTAL CONCENTRATIONS IN SOIL AND VEGETATION, 1979
}

\author{
by \\ Roger W. Ferenbaugh, Ernest S. Gladney, \\ and George H. Brooks, Jr.
}

\begin{abstract}
In 1979, soil and vegetation samples were collected on Sigma Mesa to provide background data before construction on the mesa. Elemental data are presented for soil, grass, juniper, piñon pine, and oak. None of the data looks out of the ordinary.
\end{abstract}

\section{INTRODUCTION}

In 1979, Sigma Mesa was a relatively undisturbed area east of the buildings located immediately adjacent to Diamond Drive. At that time, the only activity that had occurred on the mesa at any distance from Diamond Drive was the erection of an antenna farm about 1 mile from the road. In 1979, the decision was made to drill a geothermal well on Sigma Mesa. This activity was scheduled to begin in the summer of 1979 . Sigma Mesa also was projected to be a growth area for contractor facilities. For these reasons, a project was initiated in the Environmental Surveillance Group (H-8, renamed the Environmental Protection Group [HSE-8] in 1989) to undertake a comprehensive soil and vegetation sampling program on Sigma Mesa. The purpose of the sampling program was to acquire, before any disturbance, a set of data to be used as background for future impact analysis.

\section{METHODS}

A sampling grid was established using the proposed location of the geothermal well as the center of the grid.
Transects were run at eight compass points: $N, N E, E$, SE, S, SW, W, and NW. Samples were collected along these transects at $250 \mathrm{ft}, 500 \mathrm{ft}$, and thereafter at intervals of $500 \mathrm{ft}$ out to $2500 \mathrm{ft}$, or as far as possible before the transect was terminated because of obstacles (such as mesa walls and roads). Table I gives a tabulation of sampling sites and samples collected at each site. Figure 1 shows the location of the sampling area within Laboratory boundaries, and Fig. 2 shows individual sampling locations.

At each location, surface soil samples (0 to 2 in. deep) and grass samples were collected. Foliage samples were collected if tree species were present. The three tree species that were encountered were oak (Quercus undulata), piñon pine (Pinus edulis), and juniper (Juniperus monosperma). Grasses were not identified as to species.

Soil samples were passed through a coarse sieve (20 mesh) to remove matter such as pebbles and twigs, and then the samples were air-dried and ground in a Spex Industries shatterbox. Vegetation samples were dried in a 


\begin{tabular}{|c|c|c|c|c|c|c|}
\hline \multirow{2}{*}{$\begin{array}{l}\text { Sample } \\
\text { Designation }\end{array}$} & \multirow[b]{2}{*}{ Location } & \multicolumn{5}{|c|}{ Type of Sample Collected } \\
\hline & & Soil & Grass & Juniper & Piñon & Oak \\
\hline $\begin{array}{l}1 N \\
2 N \\
3 N \\
4 N \\
5 N\end{array}$ & $\begin{array}{r}250 \mathrm{ft} \\
500 \mathrm{ft} \\
1000 \mathrm{ft} \\
\text { Gravel pit } \\
\text { Above pit }\end{array}$ & $\begin{array}{l}\mathbf{X} \\
\mathbf{X} \\
\mathbf{X} \\
\mathbf{X} \\
\mathbf{X}\end{array}$ & $\begin{array}{l}\mathbf{X} \\
\mathbf{X} \\
\mathbf{X} \\
\mathbf{X} \\
\mathbf{X}\end{array}$ & $\mathbf{x}$ & $\begin{array}{l}\mathbf{X} \\
\mathbf{X} \\
\mathbf{X}\end{array}$ & $\mathbf{X}$ \\
\hline $\begin{array}{l}1 \mathrm{NE} \\
2 \mathrm{NE} \\
3 \mathrm{NE} \\
4 \mathrm{NE} \\
5 \mathrm{NE} \\
6 \mathrm{NE}\end{array}$ & $\begin{array}{r}250 \mathrm{ft} \\
500 \mathrm{ft} \\
1000 \mathrm{ft} \\
1500 \mathrm{ft} \\
2000 \mathrm{ft} \\
2640 \mathrm{ft}\end{array}$ & $\begin{array}{l}\mathbf{X} \\
\mathbf{X} \\
\mathbf{X} \\
\mathbf{X} \\
\mathbf{X} \\
\mathbf{X}\end{array}$ & $\begin{array}{l}\mathbf{X} \\
\mathbf{X} \\
\mathbf{X} \\
\mathbf{X} \\
\mathbf{X} \\
\mathbf{X}\end{array}$ & $\begin{array}{l}\mathbf{X} \\
\mathbf{X} \\
\mathbf{X}\end{array}$ & $\begin{array}{l}\mathbf{X} \\
\mathbf{X} \\
\mathbf{X}\end{array}$ & $\mathbf{X}$ \\
\hline $\begin{array}{l}1 \mathrm{E} \\
2 \mathrm{E} \\
3 \mathrm{E} \\
4 \mathrm{E} \\
5 \mathrm{E} \\
6 \mathrm{E} \\
7 \mathrm{E}\end{array}$ & $\begin{array}{c}250 \mathrm{ft} \\
500 \mathrm{ft} \\
1000 \mathrm{ft} \\
1500 \mathrm{ft} \\
2000 \mathrm{ft} \\
2640 \mathrm{ft} \\
\text { Knoll }\end{array}$ & $\begin{array}{l}\mathbf{X} \\
\mathbf{X} \\
\mathbf{X} \\
\mathbf{X} \\
\mathbf{X} \\
\mathbf{X} \\
\mathbf{X}\end{array}$ & $\begin{array}{l}\mathbf{X} \\
\mathbf{X} \\
\mathbf{X} \\
\mathbf{X} \\
\mathbf{X} \\
\mathbf{X} \\
\mathbf{X}\end{array}$ & $\begin{array}{l}\mathbf{X} \\
\mathbf{X} \\
\mathbf{X} \\
\mathbf{X} \\
\mathbf{X} \\
\\
\mathbf{X}\end{array}$ & $\mathbf{x}$ & $\mathbf{X}$ \\
\hline $\begin{array}{l}\text { ISE } \\
2 S E \\
3 S E\end{array}$ & $\begin{array}{c}250 \mathrm{ft} \\
500 \mathrm{ft} \\
\text { rA-35, above ponds }\end{array}$ & $\begin{array}{l}\mathbf{X} \\
\mathbf{X} \\
\mathbf{X}\end{array}$ & $\begin{array}{l}\mathbf{X} \\
\mathbf{X} \\
\mathbf{X}\end{array}$ & $\mathbf{X}$ & $\begin{array}{l}\mathbf{X} \\
\mathbf{X}\end{array}$ & $\begin{array}{l}\mathbf{X} \\
\mathbf{X}\end{array}$ \\
\hline $\begin{array}{l}1 S \\
2 S \\
3 S\end{array}$ & $\begin{array}{c}250 \mathrm{ft} \\
500 \mathrm{ft} \\
\mathrm{TA}-35\end{array}$ & $\begin{array}{l}\mathbf{X} \\
\mathbf{X} \\
\mathbf{X}\end{array}$ & $\begin{array}{l}\mathbf{X} \\
\mathbf{X} \\
\mathbf{X}\end{array}$ & $\mathbf{X}$ & $\begin{array}{l}\mathbf{X} \\
\mathbf{X}\end{array}$ & $\begin{array}{l}\mathbf{X} \\
\mathbf{X}\end{array}$ \\
\hline $\begin{array}{l}1 S W \\
2 S W \\
3 S W\end{array}$ & $\begin{array}{c}250 \mathrm{ft} \\
500 \mathrm{ft} \\
\text { Trailers at TA-35 }\end{array}$ & $\begin{array}{l}\mathbf{X} \\
\mathbf{X} \\
\mathbf{X}\end{array}$ & $\begin{array}{l}\mathbf{X} \\
\mathbf{X} \\
\mathbf{X}\end{array}$ & $\mathbf{X}$ & $\mathbf{X}$ & $\begin{array}{l}\mathbf{X} \\
\mathbf{X}\end{array}$ \\
\hline $\begin{array}{l}1 W \\
2 W \\
3 W \\
4 W \\
5 W \\
6 W \\
7 W\end{array}$ & $\begin{array}{c}\text { Wellhead } \\
250 \mathrm{ft} \\
500 \mathrm{ft} \\
1000 \mathrm{ft} \\
1500 \mathrm{ft} \\
2000 \mathrm{ft} \\
2640 \mathrm{ft}\end{array}$ & $\begin{array}{l}\mathbf{X} \\
\mathbf{X} \\
\mathbf{X} \\
\mathbf{X} \\
\mathbf{X} \\
\mathbf{X} \\
\mathbf{X}\end{array}$ & $\begin{array}{l}\mathbf{X} \\
\mathbf{X} \\
\mathbf{X} \\
\mathbf{X} \\
\mathbf{X} \\
\mathbf{X} \\
\mathbf{X}\end{array}$ & $\begin{array}{l}\mathbf{X} \\
\mathbf{X} \\
\mathbf{X} \\
\mathbf{X} \\
\\
\mathbf{X} \\
\mathbf{X}\end{array}$ & $\mathbf{X}$ & \\
\hline $\begin{array}{l}1 N W \\
2 N W \\
3 N W \\
4 N W \\
5 N W \\
6 N W\end{array}$ & $\begin{array}{r}250 \mathrm{ft} \\
500 \mathrm{ft} \\
1000 \mathrm{ft} \\
1500 \mathrm{ft} \\
2000 \mathrm{ft} \\
2640 \mathrm{ft}\end{array}$ & $\begin{array}{l}\mathbf{X} \\
\mathbf{X} \\
\mathbf{X} \\
\mathbf{X} \\
\mathbf{X} \\
\mathbf{X}\end{array}$ & $\begin{array}{l}\mathbf{X} \\
\mathbf{X} \\
\mathbf{X} \\
\mathbf{X} \\
\mathbf{X} \\
\mathbf{X}\end{array}$ & $\mathbf{x}$ & $\begin{array}{l}\mathbf{X} \\
\mathbf{X} \\
\mathbf{X} \\
\mathbf{X} \\
\mathbf{X}\end{array}$ & \\
\hline
\end{tabular}




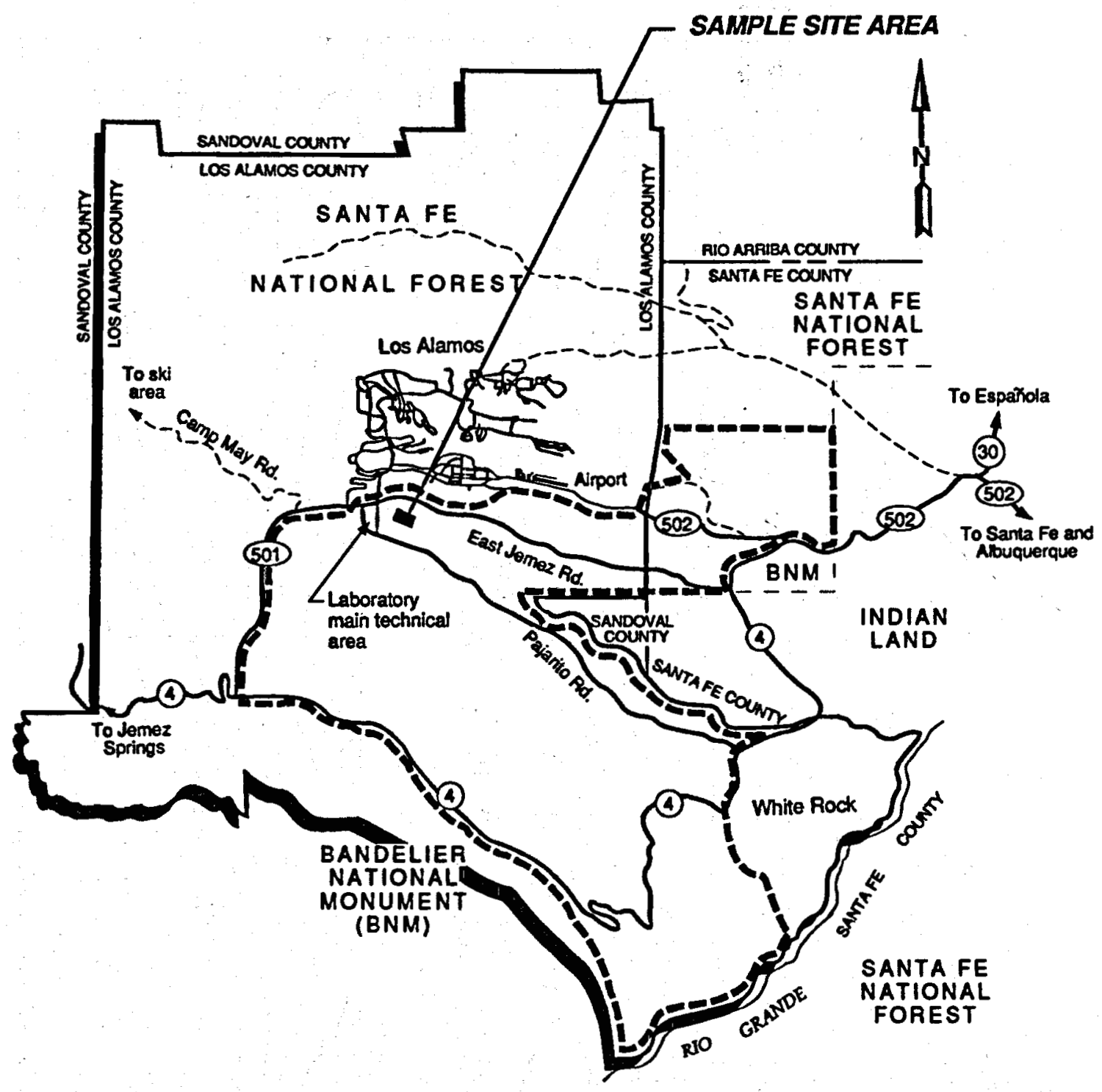

Fig. 1. Location of sampling area.

forced-air circulation oven at $60^{\circ} \mathrm{C}$ for 2 days and then were ground in the shatterbox.

After the samples were prepared, as described above, they were submitted for a variety of elemental analyses. Several analytical techniques were used, including neutron activation analysis, atomic absorption, ion chromatography, ion selective electrode analysis, and some special analytical techniques. The procedures used for these analyses have been described in detail in Gautier and Gladney (1986) and Gladney et al. (1980).
Quality assurance was provided by concurrent analysis of a variety of National Bureau of Standards (NBS), United States Environmental Protection Agency (EPA), and United States Geological Survey (USGS) reference materials using the approach documented in Gladney et al. (1981).

\section{RESULTS}

Table II summarizes the means and standard deviations of the soil data, Tables III through VI summarize 


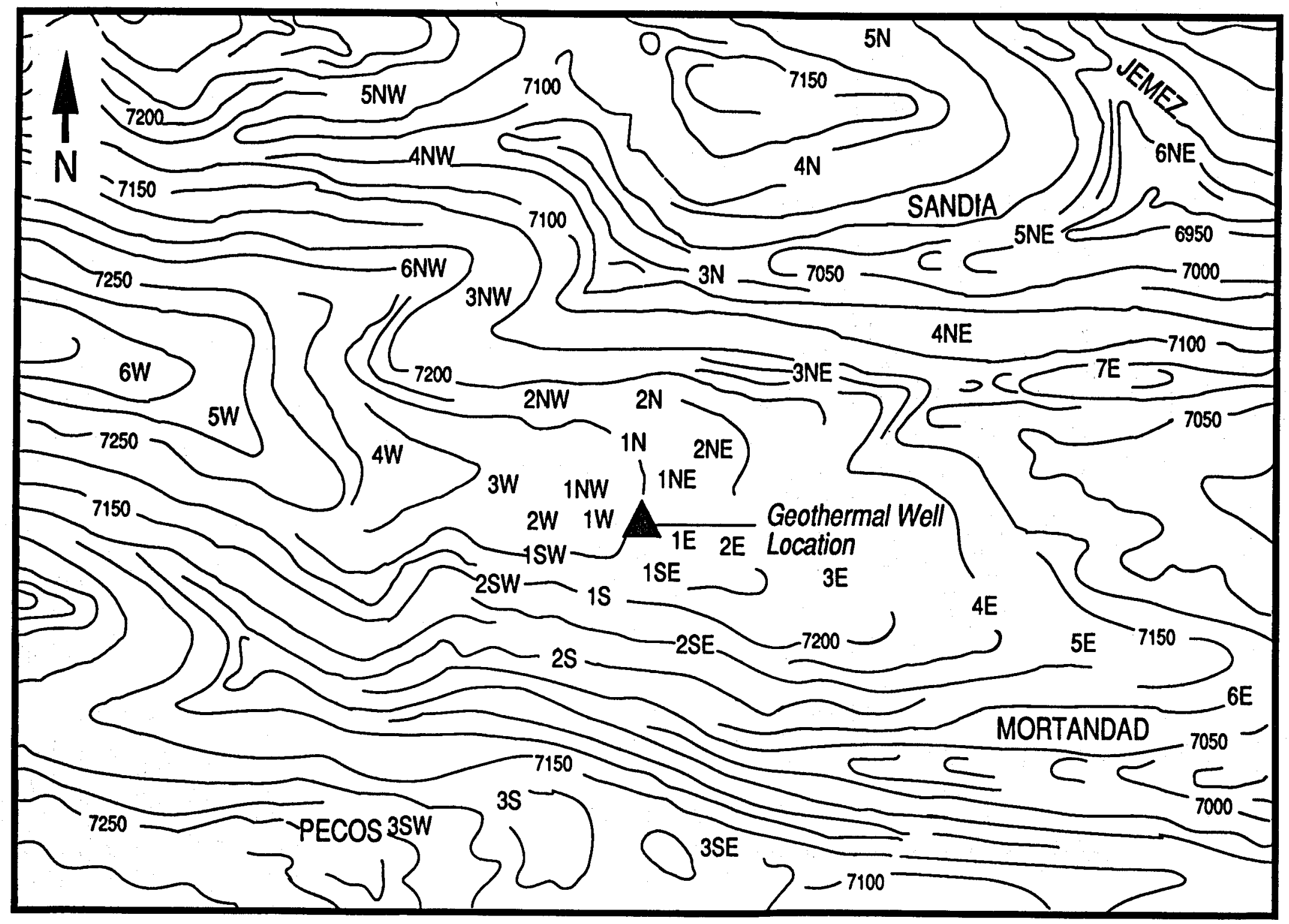

Fig. 2. Locations of sampling sites. 


\begin{tabular}{|c|c|c|c|c|c|}
\hline Element ${ }^{a}$ & Mean & $\begin{array}{l}\text { Standard } \\
\text { Deviation }\end{array}$ & $\begin{array}{l}\text { No. of } \\
\text { Samples }\end{array}$ & Maximum & Minimum \\
\hline $\mathrm{Al}(\%)$ & 5.8 & 0.35 & 40 & 6.7 & 5.3 \\
\hline As & 3.9 & 1.6 & 40 & 6.7 & 1.3 \\
\hline B & 16 & 7.2 & 38 & 27 & 7.0 \\
\hline $\mathbf{B a}$ & 410 & 220 & 40 & 810 & 120 \\
\hline $\mathrm{Be}$ & 1.9 & 0.49 & 37 & 3.3 & 1.1 \\
\hline $\mathrm{Br}$ & 1.9 & 1.2 & 38 & 5.7 & 0.40 \\
\hline Cd (ppb) & 170 & 100 & 36 & 520 & 30 \\
\hline $\mathbf{C l}$ & $<100$ & & 40 & & \\
\hline $\mathrm{Cr}$ & 27 & 24 & 40 & 136 & 4.2 \\
\hline $\mathrm{Cu}$ & 10 & 4.5 & 40 & 18 & 2.0 \\
\hline F & 240 & 74 & 40 & 390 & 50 \\
\hline $\mathrm{Fe}(\%)$ & 1.7 & 0.48 & 40 & 2.6 & 1.0 \\
\hline $\mathrm{Hg}(\mathrm{ppb})$ & 18 & 6.0 & 39 & 29 & 7.0 \\
\hline $\mathbf{L i}$ & 24 & 4.6 & 40 & 39 & 19 \\
\hline $\operatorname{Mg}(\%)$ & 0.23 & 0.12 & 40 & 0.40 & 0.051 \\
\hline $\mathbf{M n}$ & 510 & 130 & 40 & 840 & 330 \\
\hline $\mathbf{N i}$ & 8.9 & 4.8 & 40 & 19 & 1.6 \\
\hline $\mathrm{NO}_{3}$ & 8.1 & 6.5 & 30 & 26 & 0.50 \\
\hline $\mathrm{Pb}$ & 24 & 15 & 40 & 98 & 8.0 \\
\hline $\mathrm{PO}_{4}$ & 11 & 20 & 21 & 94 & 0.10 \\
\hline $\mathbf{R b}$ & 120 & 15 & 40 & 160 & 90 \\
\hline $\mathrm{SO}_{4}$ & 10 & 13 & 39 & 59 & 2.0 \\
\hline $\operatorname{Ti}(\%)$ & 0.26 & 0.15 & 40 & 0.49 & 0.079 \\
\hline $\mathrm{Zn}$ & 54 & 12 & 40 & 71 & 38 \\
\hline
\end{tabular}

vegetation data, and Table VII shows soil data from other sources for comparison with the data in Table II. In general, the Sigma Mesa data agree well with the data from other sources. Those instances where there is some discrepancy can be attributed to the chemical characteris- tics of the volcanic tuff from which the Sigma Mesa soil is derived.

The results of the individual analyses are tabulated in the Appendix, Tables A-I through A-V. 


\begin{tabular}{|c|c|c|c|c|c|}
\hline Element ${ }^{a}$ & Mean & $\begin{array}{l}\text { Standard } \\
\text { Deviation }\end{array}$ & $\begin{array}{c}\text { No. of } \\
\text { Samples }\end{array}$ & Maximum & Minimum \\
\hline $\mathrm{Al}$ & 650 & 770 & 39 & 4400 & 150 \\
\hline As (ppb) & 360 & 210 & 38 & 960 & 60 \\
\hline B & 14 & 7.0 & 29 & 34 & 7.0 \\
\hline $\mathrm{Ba}$ & 73 & 50 & 15 & 200 & 13 \\
\hline $\mathrm{Be}(\mathrm{ppb})$ & 12 & 10 & 14 & 42 & 4.0 \\
\hline $\mathrm{Br}$ & 40 & 41 & 40 & 160 & 2.7 \\
\hline $\mathrm{Cd}$ & $<200$ & & 15 & & \\
\hline $\mathrm{Cl}(\%)$ & 0.19 & 0.12 & 40 & 0.60 & 0.034 \\
\hline $\mathrm{Cr}$ & 5.5 & 3.2 & 40 & 13 & 1.6 \\
\hline $\mathrm{Cu}$ & 6.8 & 3.0 & 16 & 14 & 3.3 \\
\hline $\mathbf{F}$ & 1.1 & 0.60 & 40 & 3.4 & 0.60 \\
\hline $\mathrm{Fe}$ & 260 & 190 & 40 & 810 & 60 \\
\hline Li (ppb) & 40 & 190 & 11 & 750 & 200 \\
\hline $\mathrm{Mg}(\%)$ & 0.12 & 0.028 & 16 & 0.17 & 0.061 \\
\hline Mn & 48 & 35 & 40 & 180 & 13 \\
\hline $\mathbf{N i}$ & 26 & 11 & 15 & 55 & 7.0 \\
\hline $\mathrm{NO}_{3}$ & 420 & 380 & 37 & 1300 & 60 \\
\hline $\mathrm{Pb}$ & 1.7 & 1.1 & 11 & 4.0 & 1.0 \\
\hline $\mathrm{PO}_{4}(\%)$ & 0.19 & 0.083 & 40 & 0.47 & 0.070 \\
\hline $\mathbf{R b}$ & 5.6 & 3.2 & 37 & 18 & 2.1 \\
\hline $\mathrm{SO}_{4}$ & 690 & 360 & 40 & 1500 & 120 \\
\hline $\mathrm{Ti}$ & 46 & 60 & 15 & 250 & 12 \\
\hline $\mathbf{Z n}$ & 21 & 11 & 15 & 52 & 9.4 \\
\hline
\end{tabular}




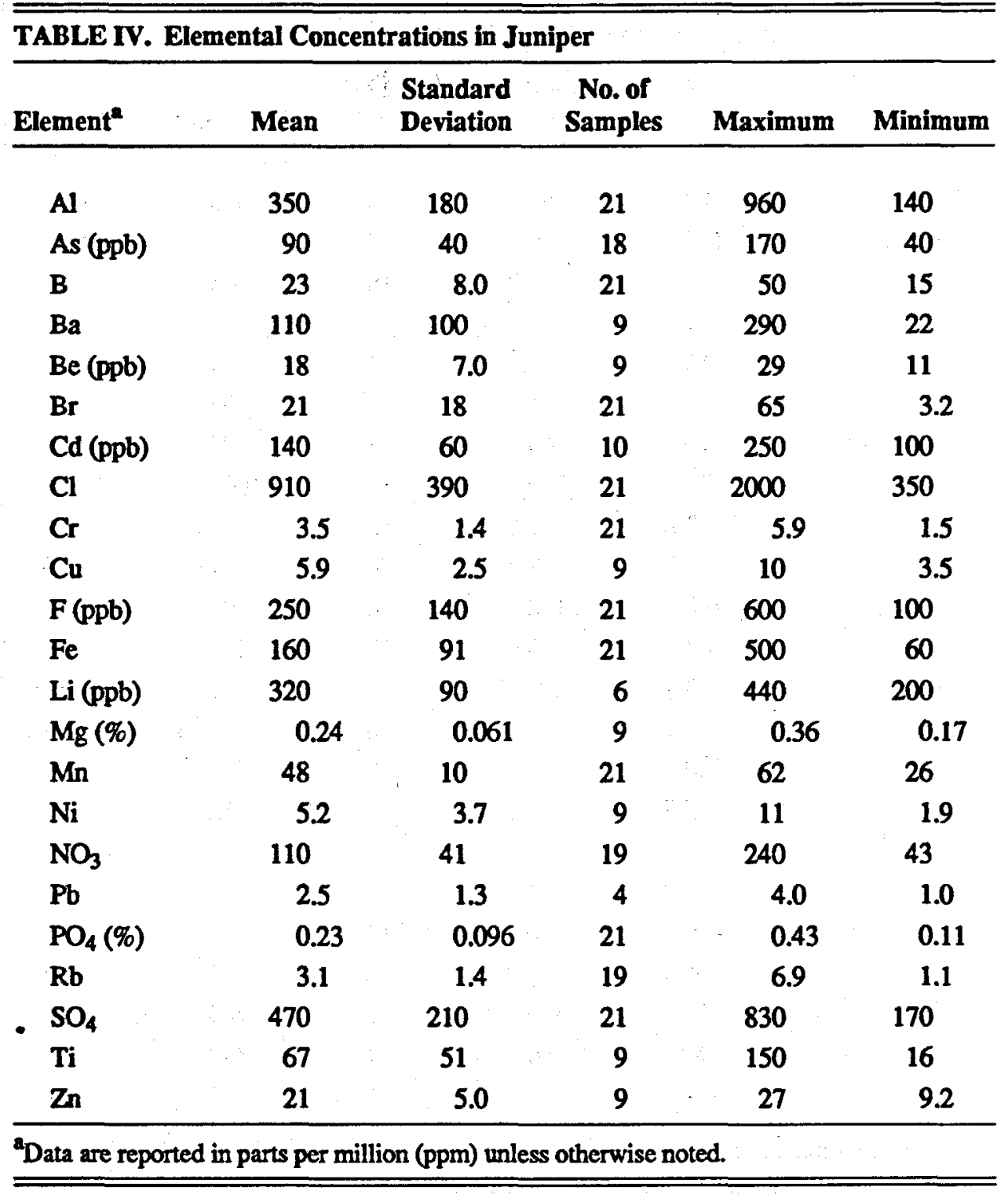




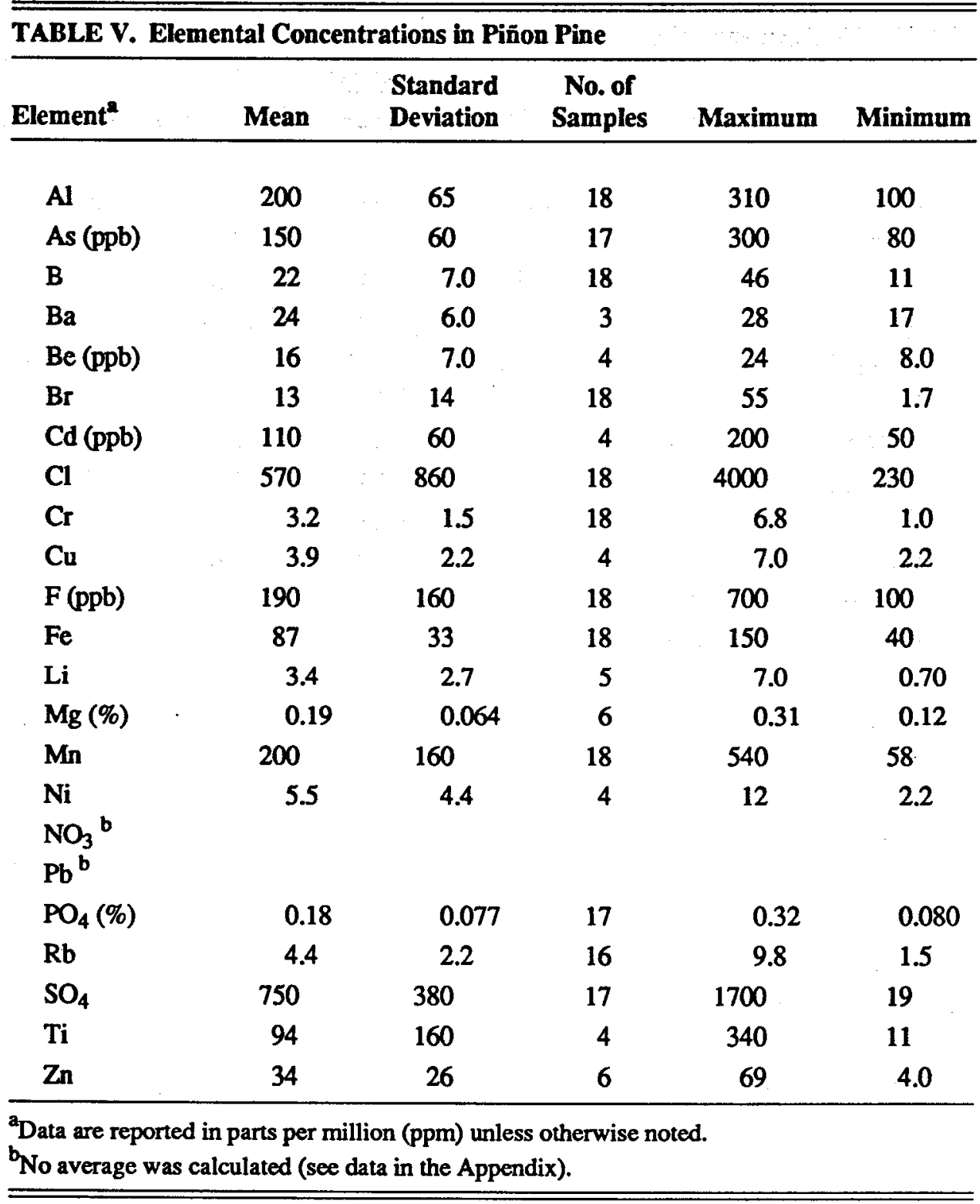




\begin{tabular}{|c|c|c|c|c|c|}
\hline Element ${ }^{2}$ & Mean & $\begin{array}{l}\text { Standard } \\
\text { Deviation }\end{array}$ & $\begin{array}{l}\text { No. of } \\
\text { Samples }\end{array}$ & Maximum & Minimum \\
\hline $\mathrm{Al}$ & 510 & 220 & 9 & 860 & 210 \\
\hline As (ppb) & 170 & 110 & 9 & 440 & 70 \\
\hline B & 63 & 13 & 9 & 81 & 48 \\
\hline $\mathbf{B a}$ & 39 & 30 & 3 & 73 & 18 \\
\hline $\mathrm{Be}(\mathrm{ppb})$ & 46 & 39 & 3 & 90 & 18 \\
\hline $\mathrm{Br}$ & 6.4 & 4.6 & 9 & 16 & 2.2 \\
\hline$C d(p p b)$ & $<200$ & & 3 & & \\
\hline $\mathbf{C l}$ & 260 & 92 & 9 & 380 & 65 \\
\hline $\mathrm{Cr}$ & 4.0 & 2.0 & 9 & 6.9 & 1.9 \\
\hline $\mathrm{Cu}$ & 7.0 & 1.3 & 3 & 8.1 & 5.5 \\
\hline F (ppb) & 260 & 150 & 9 & 600 & 100 \\
\hline $\mathrm{Fe}$ & 210 & 68 & 9 & 350 & 140 \\
\hline $\mathbf{L i}$ & 4.3 & 3.5 & 3 & 7.1 & 0.31 \\
\hline $\mathrm{Mg}(\%)$ & 0.25 & 0.026 & 3 & 0.28 & 0.23 \\
\hline $\mathbf{M n}$ & 500 & 180 & 9 & 870 & 220 \\
\hline $\mathbf{N i}$ & 4.4 & 0.40 & 3 & 4.8 & 4.0 \\
\hline $\mathrm{NO}_{3}$ & 120 & 34 & 7 & 190 & 80 \\
\hline $\mathbf{P b}$ & $<3.0$ & & 3 & & \\
\hline $\mathrm{PO}_{4}(\%)$ & 0.17 & 0.076 & 9 & 0.28 & 0.019 \\
\hline $\mathbf{R b}$ & 17 & 10 & 3 & 26 & 5.9 \\
\hline $\mathrm{SO}_{4}$ & 470 & 320 & 8 & 990 & 200 \\
\hline $\mathbf{T i}$ & 29 & 5.0 & 3 & 34 & 24 \\
\hline $\mathbf{Z n}$ & 25 & 6.0 & 3 & 30 & 18 \\
\hline
\end{tabular}




\begin{tabular}{|c|c|c|c|c|}
\hline \multirow[b]{2}{*}{ Element } & \multicolumn{4}{|c|}{ Concentrations (ppm) } \\
\hline & Mason & Vinogradov & Vinogradov & Wedepohl $^{d}$ \\
\hline Al & 81300 & 104500 & 71300 & 78300 \\
\hline As & 1.8 & 6.6 & 5 & 1.7 \\
\hline $\mathrm{Ba}$ & 425 & 800 & 500 & 590 \\
\hline $\mathrm{Be}$ & 2.8 & 7 & 6 & 2 \\
\hline $\mathrm{Br}$ & 2.5 & 6 & 5 & 2.9 \\
\hline Cd & 0.2 & 0.3 & 0.5 & 0.1 \\
\hline $\mathrm{Cl}$ & 130 & 160 & 100 & 320 \\
\hline $\mathrm{Cr}$ & 100 & 160 & 200 & 70 \\
\hline $\mathrm{Cu}$ & 55 & 57 & 20 & 30 \\
\hline $\mathbf{F}$ & 625 & 500 & 200 & 720 \\
\hline $\mathrm{Fe}$ & 50000 & 33300 & 38000 & 35400 \\
\hline $\mathrm{Hg}$ & 0.08 & 0.4 & 0.01 & 0.03 \\
\hline $\mathbf{L i}$ & 20 & 60 & 30 & 30 \\
\hline $\mathbf{M g}$ & 20900 & 13400 & 6300 & 13900 \\
\hline Mn & 950 & 670 & 850 & 690 \\
\hline $\mathrm{Ni}$ & 75 & 95 & 40 & 44 \\
\hline $\mathrm{Pb}$ & 13 & 20 & 10 & 15 \\
\hline $\mathbf{R b}$ & 90 & 400 & 100 & 120 \\
\hline $\mathrm{Ti}$ & 4400 & 4500 & 4600 & 4700 \\
\hline $\mathbf{Z n}$ & 70 & 80 & 50 & 60 \\
\hline $\mathrm{Ag}$ & 0.07 & 0.9 & 0.1 & 0.06 \\
\hline $\mathrm{Au}$ & 0.004 & - & - & 0.004 \\
\hline $\mathrm{Ca}$ & 36300 & 25300 & 13700 & 28700 \\
\hline $\mathrm{Ce}$ & 60 & 30 & 50 & 75 \\
\hline Co & 25 & 23 & 8 & 12 \\
\hline $\mathrm{Cs}$ & 3 & 12 & 5 & 2.7 \\
\hline Dy & 3 & 4 & - & 6.1 \\
\hline Eu & 1.2 & 1 & - & 1.4 \\
\hline $\mathrm{Ga}$ & 15 & 40 & 30 & 17 \\
\hline Gd & 5.4 & 5 & - & 8 \\
\hline $\mathrm{Ge}$ & 1.5 & 7 & 1 & 1.3 \\
\hline Hf & 3 & 4 & 6 & 3 \\
\hline I & 0.5 & 1 & 5 & 0.5 \\
\hline In & 0.1 & - & - & 0.07 \\
\hline $\mathbf{K}$ & 25900 & 22800 & 13600 & 28200 \\
\hline La & 30 & 40 & 40 & 44 \\
\hline Lu & 0.5 & 0.2 & - & 0.6 \\
\hline Mo & 1.5 & 2 & 2 & 1 \\
\hline $\mathrm{Na}$ & 28300 & 6600 & 6300 & 24500 \\
\hline $\mathbf{N b}$ & 20 & 20 & - & 20 \\
\hline Nd & 28 & 18 & - & $>30$ \\
\hline $\mathbf{S}$ & 260 & 3000 & 850 & 310 \\
\hline $\mathbf{S b}$ & 0.2 & 1 & - & 0.2 \\
\hline $\mathrm{Sc}$ & 22 & 10 & 7 & 14 \\
\hline $\mathrm{Se}$ & 0.05 & 0.6 & - & 0.09 \\
\hline $\mathbf{S i}$ & 277000 & 248000 & 330000 & 305000 \\
\hline
\end{tabular}


TABLE VI (Continued)

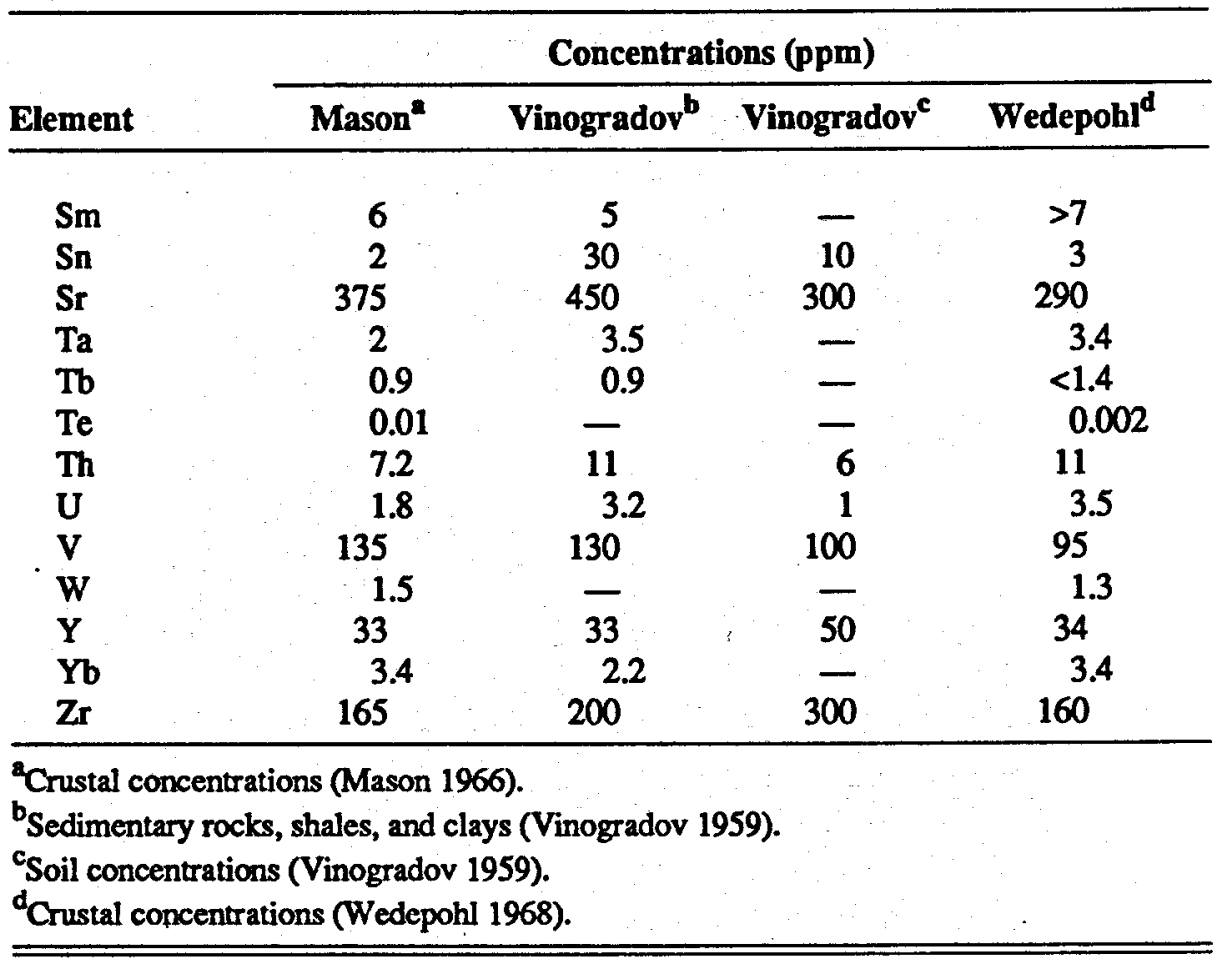

\section{REFERENCES}

Gautier, M. A., and E. S. Gladney, Eds., "Health and Environmental Chemistry: Analytical Techniques, Data Management, and Quality Assurance," Los Alamos National Laboratory report LA-10300-M (1986), Vols. I and II.

Gladney, E. S., D. B. Curtis, D. R. Perrin, J. W. Owens, and W.E. Goode, "Nuclear Techniques for the Chemical Analysis of Environmental Materials," Los Alamos Scientific Laboratory report LA-8192-MS (1980).

Gladney, E. S., J. W. Owens, T. C. Gunderson, and W. E. Goode, "Quality Assurance for Environmental
Analytical Chemistry: 1976-1979," Los Alamos National Laboratory report LA-8730-MS (1981).

Mason, B. J., Introduction to Geochemistry, 3d ed. (John Wiley \& Sons, Inc., New York, 1966).

Vinogradov, A. P., The Geochemistry of Rare and Dispersed Chemical Elements in Soils, English translation, 2d ed. (Consultants. Bureau, New York, 1959).

Wedepohl, K. H., Origin and Distribution of the Elements, L. H. Ahrens, Ed. (Pergamon Press, London, 1968), pp. 999-1016. 
APPENDIX 
TABLE A-I. Soil: Elemental Concentrations

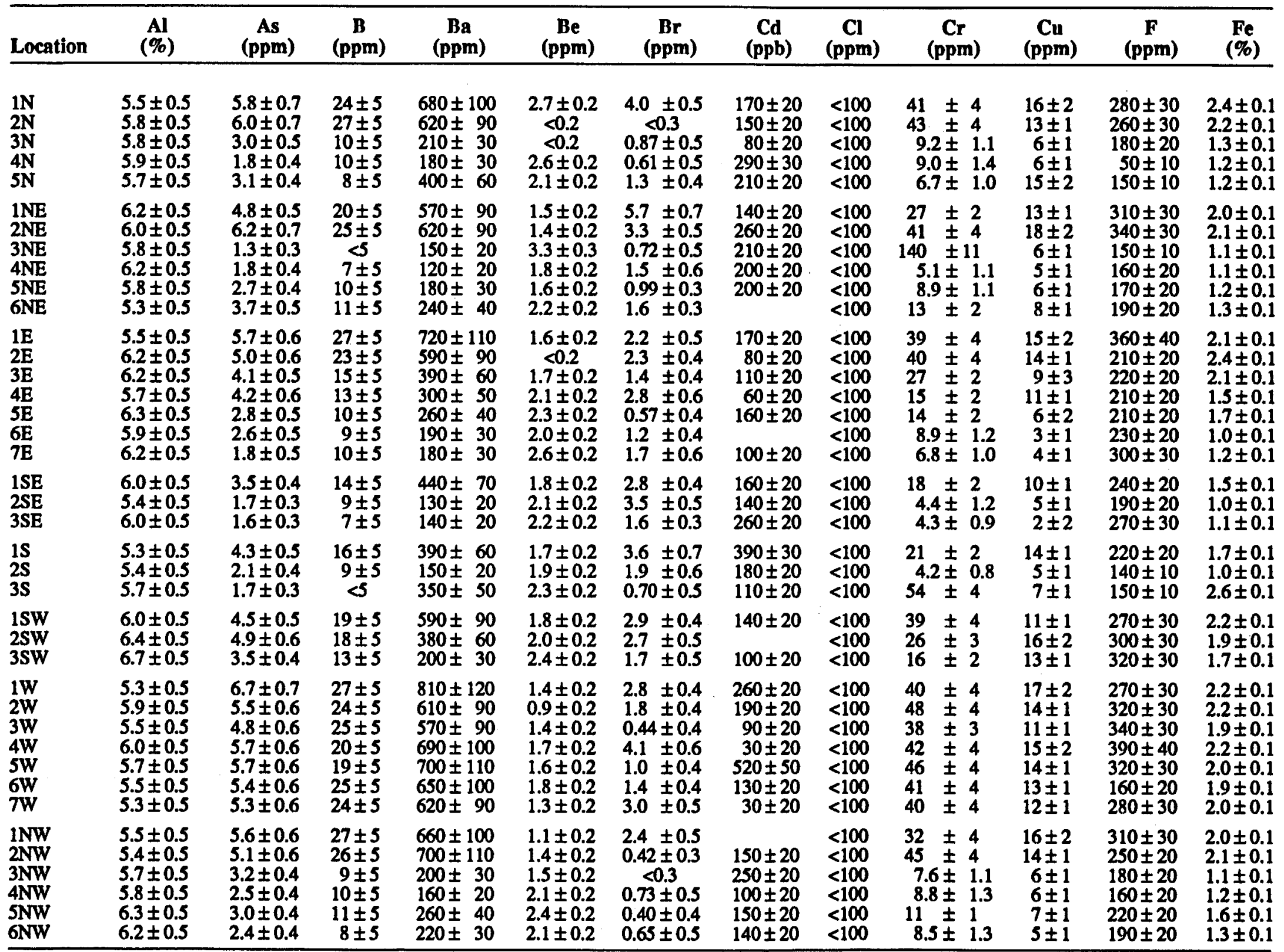




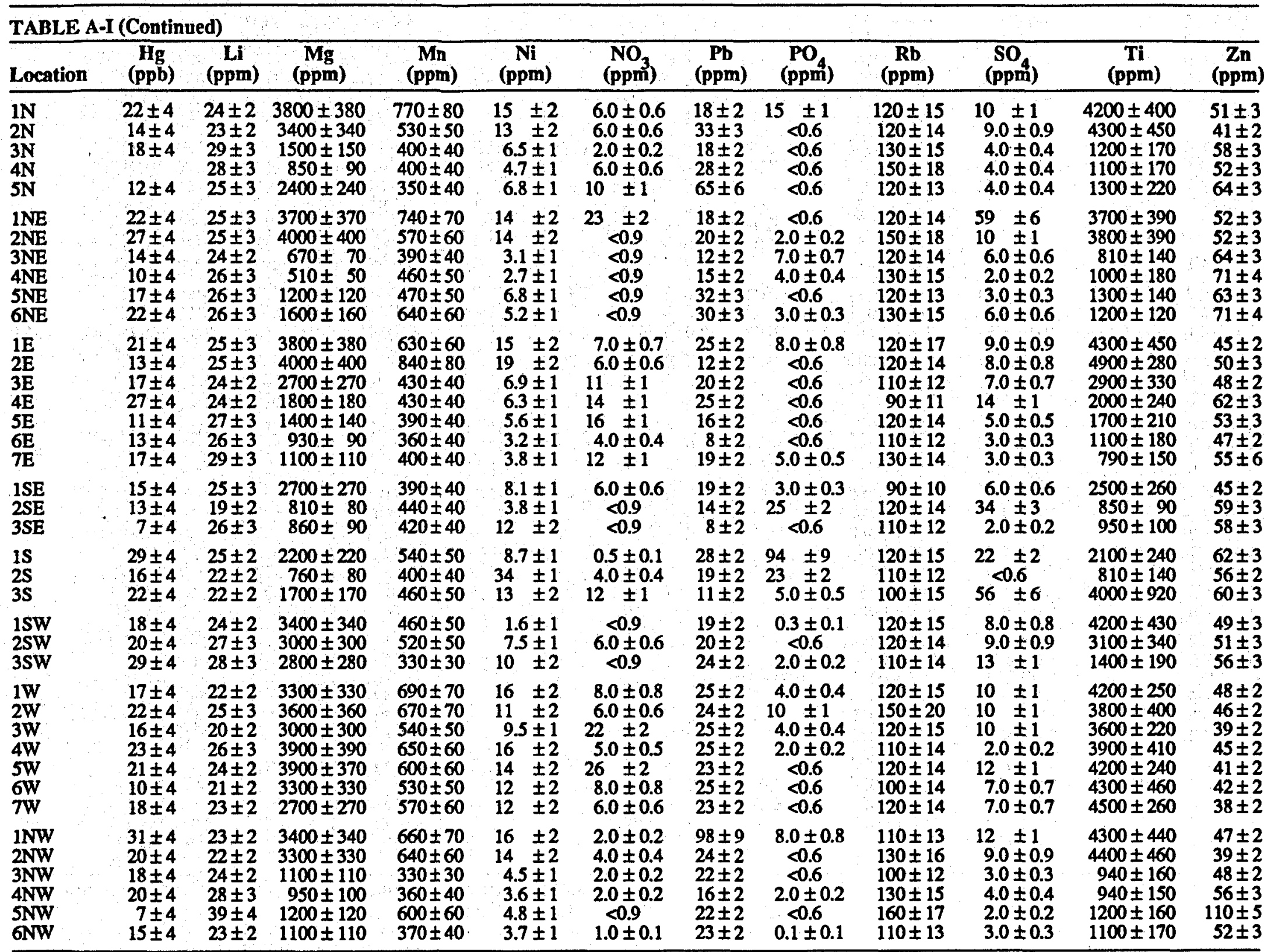

in ansufficient sample for analysis where no data are reported. Uncertainties represent analytical uncertainties. 
TABLE A-H. Grass: Elemental Concentrations ${ }^{a}$

\begin{tabular}{|c|c|c|c|c|c|c|c|c|c|c|c|c|c|}
\hline \multirow{2}{*}{$\begin{array}{l}\text { Location } \\
1 N \\
2 N \\
3 N \\
4 N \\
5 N\end{array}$} & \multicolumn{2}{|c|}{$\underset{(\mathbf{p p m})}{\mathrm{Al}}$} & \multirow{2}{*}{ 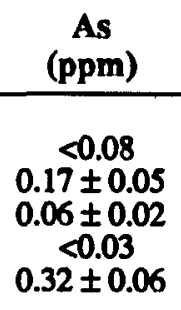 } & \multirow{2}{*}{$\begin{array}{c}\begin{array}{c}B \\
(\mathrm{ppm})\end{array} \\
\\
<10 \\
22 \pm 5 \\
7 \pm 5 \\
6 \pm 5 \\
14 \pm 5\end{array}$} & \multirow{2}{*}{$\begin{array}{c}\begin{array}{c}\text { Ba } \\
(\mathrm{ppm})\end{array} \\
\\
44 \pm 10 \\
90 \pm 20\end{array}$} & \multirow{2}{*}{$\begin{array}{c}\begin{array}{c}\text { Be } \\
(\mathbf{p p b})\end{array} \\
\\
\\
21 \pm 2 \\
11 \pm 2\end{array}$} & \multicolumn{2}{|c|}{$\underset{(\mathbf{p p m})}{\mathbf{B r}}$} & \multirow{2}{*}{$\begin{array}{l}\begin{array}{c}\text { Cd } \\
(\mathrm{ppm})\end{array} \\
\\
<0.2 \\
<0.2\end{array}$} & \multirow{2}{*}{$\begin{array}{c}\begin{array}{c}\text { Cl } \\
(\mathrm{ppm})\end{array} \\
2700 \pm 400 \\
1700 \pm 260 \\
1500 \pm 220 \\
2400 \pm 360 \\
2000 \pm 300\end{array}$} & \multirow{2}{*}{$\begin{array}{c}\begin{array}{c}\mathbf{C r} \\
(\mathbf{p p m})\end{array} \\
5.0 \pm 0.5 \\
4.3 \pm 0.4 \\
3.1 \pm 0.3 \\
4.0 \pm 0.4 \\
3.7 \pm 0.4\end{array}$} & \multirow{2}{*}{$\begin{array}{c}\begin{array}{c}\mathrm{Cu} \\
(\mathrm{ppm})\end{array} \\
\\
\\
3.3 \pm 0.3 \\
4.4 \pm 0.4\end{array}$} & \multirow{2}{*}{$\begin{array}{c}\begin{array}{c}F \\
(\text { ppm) }\end{array} \\
1.6 \pm 0.2 \\
2.6 \pm 0.3 \\
2.4 \pm 0.2 \\
2.2 \pm 0.2 \\
1.3 \pm 0.1\end{array}$} \\
\hline & $\begin{array}{r}1300 \pm \\
1500 \pm \\
230 \pm \\
4400 \pm \\
320 \pm\end{array}$ & $\begin{array}{r}380 \\
460 \\
70 \\
1300 \\
100\end{array}$ & & & & & $\begin{array}{r}160 \\
130 \\
16 \\
31 \\
20\end{array}$ & $\begin{array}{l} \pm 20 \\
\pm 10 \\
\pm 2 \\
\pm 3 \\
\pm 2\end{array}$ & & & & & \\
\hline $\begin{array}{l}1 \mathrm{NE} \\
\text { 2NE } \\
\text { 3NE } \\
4 \mathrm{NE} \\
5 \mathrm{NE} \\
6 \mathrm{NE}\end{array}$ & $\begin{array}{l}720 \pm \\
460 \pm \\
260 \pm \\
280 \pm \\
220 \pm \\
300 \pm\end{array}$ & $\begin{array}{r}220 \\
140 \\
80 \\
90 \\
70 \\
90\end{array}$ & $\begin{array}{l}0.57 \pm 0.10 \\
0.25 \pm 0.06 \\
0.55 \pm 0.08 \\
0.23 \pm 0.05 \\
0.51 \pm 0.07 \\
0.53 \pm 0.08\end{array}$ & $\begin{array}{c}<5 \\
8 \pm 5 \\
23 \pm 5 \\
<5 \\
9 \pm 5 \\
8 \pm 5\end{array}$ & & & $\begin{array}{r}110 \\
32 \\
10 \\
11 \\
10 \\
13\end{array}$ & $\begin{array}{l} \pm 10 \\
\pm 3 \\
\pm 1 \\
\pm 1 \\
\pm 1 \\
\pm 1\end{array}$ & $0.07 \pm 0.01$ & $\begin{array}{r}2400 \pm 360 \\
1500 \pm 220 \\
900 \pm 140 \\
1500 \pm 230 \\
2600 \pm 380 \\
1500 \pm 230\end{array}$ & $\begin{array}{r}7.5 \pm 0.8 \\
13 \pm 1.3 \\
9.7 \pm 1.0 \\
6.1 \pm 0.6 \\
6.5 \pm 0.7 \\
4.3 \pm 0.4\end{array}$ & & $\begin{array}{l}0.8 \pm 0.1 \\
0.9 \pm 0.1 \\
0.7 \pm 0.1 \\
0.6 \pm 0.1 \\
0.6 \pm 0.1 \\
0.7 \pm 0.1\end{array}$ \\
\hline $\begin{array}{l}1 \mathbf{E} \\
2 \mathbf{E} \\
3 \mathbf{E} \\
4 \mathbf{E} \\
5 \mathbf{E} \\
6 \mathbf{E} \\
7 \mathbf{E}\end{array}$ & $\begin{array}{r}1300 \pm \\
310 \pm \\
2000 \pm \\
240 \pm \\
290 \pm \\
150 \pm \\
250 \pm\end{array}$ & $\begin{array}{r}380 \\
90 \\
610 \\
70 \\
90 \\
50 \\
80\end{array}$ & $\begin{array}{l}0.17 \pm 0.06 \\
0.08 \pm 0.05 \\
0.15 \pm 0.07 \\
0.50 \pm 0.07 \\
0.19 \pm 0.07 \\
0.31 \pm 0.05 \\
0.61 \pm 0.08\end{array}$ & $\begin{array}{c}<5 \\
<5 \\
13 \pm 5 \\
11 \pm 5 \\
<5 \\
10 \pm 5 \\
10 \pm 5\end{array}$ & $50 \pm 10$ & $4 \pm 2$ & $\begin{array}{c}51 \\
34 \\
29 \\
12 \\
50 \\
6.2 \\
13\end{array}$ & $\begin{array}{ll} \pm & 5 \\
\pm & 3 \\
\pm & 3 \\
\pm & 1 \\
\pm & 5 \\
\pm & 0.6 \\
\pm & 1\end{array}$ & $\begin{array}{l}<0.2 \\
<0.2\end{array}$ & $\begin{array}{r}1400 \pm 210 \\
960 \pm 140 \\
2100 \pm 310 \\
980 \pm 150 \\
2700 \pm 400 \\
1300 \pm 190 \\
1400 \pm 220\end{array}$ & $\begin{array}{l}3.9 \pm 0.4 \\
2.1 \pm 0.2 \\
9.6 \pm 1.0 \\
2.1 \pm 0.2 \\
2.5 \pm 0.3 \\
1.6 \pm 0.2 \\
3.5 \pm 0.4\end{array}$ & $\begin{array}{l}5.0 \pm 0.5 \\
5.0 \pm 0.5\end{array}$ & $\begin{array}{l}0.9 \pm 0.1 \\
1.5 \pm 0.1 \\
1.6 \pm 0.2 \\
1.1 \pm 0.1 \\
0.8 \pm 0.1 \\
0.8 \pm 0.1 \\
1.0 \pm 0.1\end{array}$ \\
\hline $\begin{array}{l}1 S \\
2 S \\
3 S\end{array}$ & $\begin{array}{l}290 \pm \\
250 \pm \\
240 \pm\end{array}$ & $\begin{array}{l}90 \\
70 \\
70\end{array}$ & $\begin{array}{l}0.19 \pm 0.04 \\
0.27 \pm 0.04 \\
0.39 \pm 0.06\end{array}$ & $\begin{array}{c}12 \pm 5 \\
10 \pm 5 \\
<5\end{array}$ & $90 \pm 20$ & $<3$ & $\begin{array}{l}13 \\
25 \\
15\end{array}$ & $\begin{array}{l} \pm 1 \\
\pm 2 \\
\pm 2\end{array}$ & $<0.3$ & $\begin{array}{l}1100 \pm 160 \\
1600 \pm 240 \\
6000 \pm 890\end{array}$ & $\begin{array}{r}3.9 \pm 0.4 \\
3.5 \pm 0.4 \\
13 \pm 1.3\end{array}$ & $6.9 \pm 0.7$ & $\begin{array}{l}0.6 \pm 0.1 \\
0.7 \pm 0.1 \\
0.7 \pm 0.1\end{array}$ \\
\hline $\begin{array}{l}1 S W \\
2 S W \\
3 S W\end{array}$ & $\begin{array}{l}460 \pm \\
250 \pm \\
240 \pm\end{array}$ & $\begin{array}{r}140 \\
70 \\
70\end{array}$ & $\begin{array}{l}0.31 \pm 0.05 \\
0.29 \pm 0.05 \\
0.31 \pm 0.07\end{array}$ & $\begin{array}{c}21 \pm 5 \\
9 \pm 5 \\
<5\end{array}$ & $\begin{array}{r}22 \pm 10 \\
45 \pm 10 \\
150 \pm 30\end{array}$ & $\begin{array}{l}6 \pm 2 \\
6 \pm 2 \\
5 \pm 2\end{array}$ & $\begin{array}{c}3.9 \\
19 \\
23\end{array}$ & $\begin{array}{l} \pm 0.4 \\
\pm 2 \\
\pm 2\end{array}$ & $\begin{array}{l}<0.2 \\
<0.2 \\
<0.2\end{array}$ & $\begin{array}{r}340 \pm 50 \\
1300 \pm 200 \\
5600 \pm 850\end{array}$ & $\begin{array}{r}3.9 \pm 0.4 \\
3.5 \pm 0.4 \\
13^{ \pm} \pm 1.3\end{array}$ & $\begin{array}{l}9.2 \pm 0.9 \\
7.6 \pm 0.8 \\
3.9 \pm 0.4\end{array}$ & $\begin{array}{l}1.2 \pm 0.1 \\
0.7 \pm 0.1 \\
0.6 \pm 0.1\end{array}$ \\
\hline $\begin{array}{l}1 W \\
2 W \\
3 W \\
4 W \\
5 W \\
6 W \\
7 W\end{array}$ & $\begin{array}{r}1500 \pm \\
600 \pm \\
1100 \pm \\
810 \pm \\
1200 \pm \\
710 \pm\end{array}$ & $\begin{array}{l}460 \\
180 \\
340 \\
240 \\
350 \\
210\end{array}$ & $\begin{array}{l}0.81 \pm 0.12 \\
0.37 \pm 0.11 \\
0.72 \pm 0.13 \\
0.48 \pm 0.10 \\
0.96 \pm 0.14 \\
0.59 \pm 0.10 \\
0.20 \pm 0.06\end{array}$ & $\begin{array}{l}34 \pm 5 \\
<5 \\
12 \pm 5 \\
26 \pm 5 \\
12 \pm 5 \\
29 \pm 5 \\
11 \pm 5\end{array}$ & $\begin{array}{r}100 \pm 20 \\
44 \pm 10\end{array}$ & $\begin{array}{l}10 \pm 2 \\
10 \pm 2\end{array}$ & $\begin{array}{r}77 \\
98 \\
150 \\
69 \\
91 \\
72 \\
37\end{array}$ & $\begin{array}{l} \pm 8 \\
\pm 10 \\
\pm 15 \\
\pm 7 \\
\pm 9 \\
\pm 7 \\
\pm 4\end{array}$ & $\begin{array}{l}<0.5 \\
<0.2\end{array}$ & $\begin{array}{r}1600 \pm 250 \\
1900 \pm 280 \\
4200 \pm 630 \\
940 \pm 140 \\
2300 \pm 340 \\
2400 \pm 370 \\
2000 \pm 300\end{array}$ & $\begin{array}{r}6.9 \pm 0.7 \\
4.1 \pm 0.4 \\
5.9 \pm 0.6 \\
3.2 \pm 0.3 \\
13 \pm 1.3 \\
2.1 \pm 0.2 \\
5.1 \pm 0.5\end{array}$ & $\begin{array}{r}3.4 \pm 0.3 \\
6.8 \pm 0.7 \\
9.7 \pm 1.0 \\
11 \pm 1.1 \\
7.6 \pm 0.8\end{array}$ & $\begin{array}{l}3.4 \pm 0.3 \\
1.3 \pm 0.1 \\
1.2 \pm 0.1 \\
1.6 \pm 0.2 \\
1.5 \pm 0.1 \\
1.0 \pm 0.1 \\
0.8 \pm 0.1\end{array}$ \\
\hline $\begin{array}{l}1 \mathrm{NW} \\
2 \mathrm{NW} \\
3 \mathrm{NW} \\
4 \mathrm{NW} \\
5 \mathrm{NW} \\
6 \mathrm{NW}\end{array}$ & $\begin{array}{l}880 \pm \\
540 \pm \\
190 \pm \\
390 \pm \\
220 \pm \\
150 \pm\end{array}$ & $\begin{array}{r}260 \\
160 \\
60 \\
120 \\
70 \\
50\end{array}$ & $\begin{array}{l}0.29 \pm 0.08 \\
0.62 \pm 0.11 \\
0.13 \pm 0.04 \\
0.22 \pm 0.04 \\
0.25 \pm 0.05 \\
0.32 \pm 0.05\end{array}$ & $\begin{array}{c}7 \pm 5 \\
17 \pm 5 \\
11 \pm 5 \\
<5 \\
<5 \\
9 \pm 5\end{array}$ & & & $\begin{array}{c}47 \\
24 \\
5.9 \\
15 \\
17 \\
19\end{array}$ & $\begin{array}{l} \pm 5 \\
\pm 2 \\
\pm 0.6 \\
\pm 2 \\
\pm 2 \\
\pm 2\end{array}$ & & $\begin{array}{r}1900 \pm 280 \\
410 \pm 60 \\
1800 \pm 270 \\
970 \pm 150 \\
2300 \pm 350 \\
1900 \pm 290\end{array}$ & $\begin{array}{l}5.2 \pm 0.5 \\
6.3 \pm 0.6 \\
8.1 \pm 0.8 \\
4.3 \pm 0.4 \\
6.3 \pm 0.6 \\
2.8 \pm 0.3\end{array}$ & & $\begin{array}{l}1.1 \pm 0.1 \\
1.1 \pm 0.1 \\
0.6 \pm 0.1 \\
0.9 \pm 0.1 \\
0.7 \pm 0.1 \\
0.7 \pm 0.1\end{array}$ \\
\hline
\end{tabular}


TABLE A-II (Continued)

\begin{tabular}{|c|c|c|c|c|c|c|c|c|c|c|c|c|c|c|}
\hline \multirow{2}{*}{$\begin{array}{l}\text { Location } \\
1 N \\
2 N \\
3 N \\
4 N \\
5 N\end{array}$} & \multirow{2}{*}{$\begin{array}{c}\text { Fe } \\
\text { (ppm) } \\
490 \pm 100 \\
540 \pm 100 \\
90 \pm 20 \\
810 \pm 160 \\
130 \pm 30\end{array}$} & \multirow{2}{*}{$\begin{array}{c}\begin{array}{c}\mathbf{L i} \\
(\mathrm{ppm})\end{array} \\
\\
0.20 \pm 0.10 \\
<0.20\end{array}$} & \multirow{2}{*}{$\begin{array}{c}\begin{array}{c}\mathbf{M g} \\
(\mathbf{p p m})\end{array} \\
1300 \pm 130 \\
1200 \pm 120\end{array}$} & \multirow{2}{*}{$\begin{array}{c}\begin{array}{c}\text { Mn } \\
(p p m)\end{array} \\
52 \pm 11 \\
45 \pm 10 \\
28 \pm 6 \\
52 \pm 11 \\
17 \pm 5\end{array}$} & \multicolumn{2}{|c|}{$\begin{array}{c}\mathbf{N i} \\
(\mathbf{p p m})\end{array}$} & \multicolumn{2}{|c|}{$\begin{array}{c}\mathrm{NO}_{3} \\
(\mathrm{ppm})\end{array}$} & \multirow{2}{*}{$\begin{array}{c}\begin{array}{c}\mathbf{P b} \\
(\mathrm{ppm})\end{array} \\
\\
3.0 \pm 1.0 \\
2.0 \pm 1.0\end{array}$} & \multirow{2}{*}{$\begin{array}{c}\mathrm{PO}_{4} \\
(\mathrm{ppm})\end{array}$} & \multirow{2}{*}{$\begin{array}{c}\mathbf{R b} \\
(\mathrm{ppm})\end{array}$} & \multirow{2}{*}{$\begin{array}{c}\begin{array}{c}\mathrm{SO}_{4} \\
(\mathrm{ppm})\end{array} \\
360 \pm 40 \\
450 \pm 50 \\
990 \pm 100 \\
270 \pm 30 \\
570 \pm 60\end{array}$} & \multirow{2}{*}{$\begin{array}{c}\mathbf{T i} \\
(\mathbf{p p m})\end{array}$} & $\underset{(\mathrm{ppm})}{\mathrm{Zn}}$ \\
\hline & & & & & $\begin{array}{c}18 \\
7.0\end{array}$ & $\begin{array}{l} \pm 2 \\
\pm 1.0\end{array}$ & $\begin{array}{r}110 \\
810 \\
4 . \\
1300 \\
450\end{array}$ & $\begin{array}{l} \pm 10 \\
\pm 80 \\
\pm 0.4 \\
\pm 130 \\
\pm 50\end{array}$ & & & & & & $\begin{array}{c}9.4 \pm 0.9 \\
21 \pm 2\end{array}$ \\
\hline $\begin{array}{l}1 \mathrm{NE} \\
2 \mathrm{NE} \\
3 \mathrm{NE} \\
4 \mathrm{NE} \\
5 \mathrm{NE} \\
6 \mathrm{NE}\end{array}$ & $\begin{array}{l}300 \pm 60 \\
270 \pm 50 \\
240 \pm 50 \\
140 \pm 30 \\
100 \pm 20 \\
100 \pm 20\end{array}$ & & & $\begin{array}{l}39 \pm 8 \\
33 \pm 7 \\
73 \pm 15 \\
32 \pm 7 \\
35 \pm 7 \\
57 \pm 12\end{array}$ & & & $\begin{array}{r}200 \\
93 \\
190 \\
300 \\
200\end{array}$ & $\begin{array}{l} \pm 20 \\
\pm 9 \\
<0.9 \\
\pm 20 \\
\pm 30 \\
\pm 20\end{array}$ & & $\begin{array}{l}2600 \pm 260 \\
2100 \pm 210 \\
2000 \pm 200 \\
2000 \pm 200 \\
2000 \pm 200 \\
1300 \pm 130\end{array}$ & $\begin{array}{r}11 \pm 1.2 \\
2.2 \pm 1.2 \\
4.3 \pm 1.2 \\
6.4 \pm 1.2 \\
9.5 \pm 1.2 \\
2.6 \pm 1.2\end{array}$ & $\begin{array}{r}920 \pm 90 \\
1300 \pm 130 \\
520 \pm .50 \\
540 \pm 50 \\
470 \pm 50 \\
740 \pm 70\end{array}$ & & \\
\hline $\begin{array}{l}1 \mathrm{E} \\
2 \mathrm{E} \\
3 \mathrm{E} \\
4 \mathrm{E} \\
5 \mathrm{E} \\
6 \mathrm{E}\end{array}$ & $\begin{array}{c}460 \pm 90 \\
110 \pm 20 \\
230 \pm 40 \\
110 \pm 20 \\
110 \pm 20 \\
60 \pm 10 \\
90 \pm 20\end{array}$ & $\begin{array}{l}<0.20 \\
<0.20\end{array}$ & $1100 \pm 110$ & $\begin{array}{l}36 \pm 8 \\
24 \pm 5 \\
17 \pm 4 \\
23 \pm 5 \\
24 \pm 5 \\
31 \pm 6 \\
31 \pm 6\end{array}$ & 15 & \pm 4 & $\begin{array}{r}200 \\
100 \\
600 \\
150 \\
410 \\
250 \\
60\end{array}$ & $\begin{array}{l} \pm 20 \\
\pm 10 \\
\pm 60 \\
\pm 20 \\
\pm 40 \\
\pm 30 \\
\pm 6\end{array}$ & $1.0 \pm 1.0$ & $\begin{array}{r}1600 \pm 160 \\
1900 \pm 190 \\
1500 \pm 150 \\
1100 \pm 110 \\
700 \pm 70 \\
2000 \pm 200 \\
820 \pm 80\end{array}$ & $\begin{array}{c}5.4 \pm 1.2 \\
<1.2 \\
5.5 \pm 1.2 \\
3.6 \pm 1.2 \\
11 \pm 1.2 \\
4.2 \pm 1.2 \\
2.7 \pm 1.2\end{array}$ & $\begin{array}{r}320 \pm 30 \\
610 \pm 60 \\
1100 \pm 110 \\
820 \pm 80 \\
230 \pm 20 \\
990 \pm 100 \\
1200 \pm 120\end{array}$ & $16 \pm 3$ & $\begin{array}{l}14 \pm 1 \\
16 \pm 2\end{array}$ \\
\hline $\begin{array}{l}\text { 1SE } \\
\text { 2SE } \\
\text { 3SE }\end{array}$ & $\begin{array}{l}150 \pm 30 \\
170 \pm 30 \\
150 \pm 30\end{array}$ & $\begin{array}{l}0.50 \pm 0.05 \\
0.37 \pm 0.04 \\
0.20 \pm 0.10\end{array}$ & $\begin{array}{r}820 \pm 80 \\
1100 \pm 110 \\
1500 \pm 150\end{array}$ & $\begin{array}{l}26 \pm 5 \\
79 \pm 16 \\
32 \pm 7\end{array}$ & $\begin{array}{l}28 \\
20 \\
29\end{array}$ & $\begin{array}{l} \pm 3 \\
\pm 3 \\
\pm 3\end{array}$ & $\begin{array}{l}270 \\
260\end{array}$ & $\begin{array}{l} \pm 30 \\
\pm 30 \\
<0.9\end{array}$ & $\begin{array}{l}1.0 \pm 1.0 \\
1.0 \pm 1.0 \\
1.0 \pm 1.0\end{array}$ & $\begin{array}{l}90 \\
10 \\
10\end{array}$ & $\begin{array}{l}2.2 \pm 1.2 \\
2.6 \pm 1.2 \\
8.8 \pm 1.2\end{array}$ & $\begin{array}{r}1500 \pm 150 \\
270 \pm 30 \\
320 \pm 30\end{array}$ & $\begin{array}{l}29 \pm 3 \\
25 \pm 3 \\
17 \pm 3\end{array}$ & $\begin{array}{l}12 \pm 1 \\
22 \pm 2 \\
24 \pm 2\end{array}$ \\
\hline $\begin{array}{l}1 S \\
2 S \\
3 S\end{array}$ & $\begin{array}{r}130 \pm 20 \\
70 \pm 20 \\
200 \pm 40\end{array}$ & $<0.20$ & $1500 \pm 150$ & $\begin{array}{l}25 \pm 5 \\
30 \pm 6 \\
64 \pm 13\end{array}$ & 32 & & $\begin{array}{l}100 \\
100 \\
660\end{array}$ & $\begin{array}{l} \pm 10 \\
\pm 10 \\
\pm 70\end{array}$ & $<3.0$ & $\begin{array}{l}170 \\
=230 \\
200\end{array}$ & $\begin{array}{l}4.4 \pm 1.2 \\
3.8 \pm 1.2 \\
2.3 \pm 1.2\end{array}$ & $\begin{array}{l}840 \pm 80 \\
950 \pm 90 \\
130 \pm 10\end{array}$ & $16 \pm 3$ & $39 \pm 4$ \\
\hline $\begin{array}{l}1 S W \\
2 S W \\
3 S W\end{array}$ & $\begin{array}{l}160 \pm 30 \\
150 \pm 30 \\
150 \pm 30\end{array}$ & $\begin{array}{c}0.71 \pm 0.07 \\
0.35 \pm 0.04 \\
<0.20\end{array}$ & $\begin{array}{r}1100 \pm 110 \\
980 \pm 100 \\
1300 \pm 130\end{array}$ & $\begin{array}{r}180 \pm 35 \\
61 \pm 12 \\
60 \pm 12\end{array}$ & $\begin{array}{l}22 \\
28 \\
20\end{array}$ & $\begin{array}{l} \pm 3 \\
\pm 3 \\
\pm 3\end{array}$ & $\begin{array}{l}600 \\
180\end{array}$ & $\begin{array}{l} \pm 60 \\
<0.9 \\
\pm 20\end{array}$ & $\begin{array}{c}1.0 \pm 1.0 \\
<3.0 \\
<1.0\end{array}$ & $\begin{array}{r}80 \\
140 \\
470\end{array}$ & $\begin{array}{l}2 \\
1.2 \\
1.2\end{array}$ & $\begin{array}{l} \pm 30 \\
\pm 110 \\
\pm 60\end{array}$ & $\begin{array}{l}22 \pm 3 \\
24 \pm 3 \\
36 \pm 3\end{array}$ & $\begin{array}{l}16 \pm 2 \\
19 \pm 2 \\
52 \pm 5\end{array}$ \\
\hline $\begin{array}{l}1 W \\
2 W \\
3 W \\
4 W \\
5 W \\
6 W \\
7 W\end{array}$ & $\begin{array}{l}720 \pm 140 \\
270 \pm 50 \\
610 \pm 120 \\
350 \pm 70 \\
580 \pm 120 \\
470 \pm 90 \\
430 \pm 80\end{array}$ & $\begin{array}{l}0.27 \pm 0.10 \\
0.75 \pm 0.08 \\
0.45 \pm 0.05 \\
0.34 \pm 0.03 \\
0.28 \pm 0.03\end{array}$ & $\begin{array}{r}610 \pm 60 \\
1100 \pm 110 \\
1000 \pm 100 \\
1500 \pm 150 \\
1700 \pm 170\end{array}$ & $\begin{array}{l}87 \pm 18 \\
38 \pm 8 \\
54 \pm 11 \\
59 \pm 12 \\
41 \pm 9 \\
52 \pm 11 \\
74 \pm 15\end{array}$ & $\begin{array}{l}24 \\
25\end{array}$ & $\begin{array}{l} \pm 3 \\
\pm 3\end{array}$ & $\begin{array}{r}1200 \\
110 \\
690 \\
1100 \\
200 \\
1100 \\
310\end{array}$ & $\begin{array}{l} \pm 120 \\
\pm 10 \\
\pm 70 \\
\pm 110 \\
\pm 20 \\
\pm 110 \\
\pm 30\end{array}$ & $\begin{array}{l}<3.0 \\
3.0 \pm 1.0 \\
4.0 \pm 1.0 \\
1.0 \pm 1.0 \\
1.0 \pm 1.0\end{array}$ & $\begin{array}{l}4100 \pm 410 \\
1900 \pm 190 \\
3100 \pm 310 \\
3200 \pm 320 \\
1800 \pm 180 \\
2400 \pm 240 \\
1600 \pm 160\end{array}$ & $\begin{array}{l}8.0 \pm 1.2 \\
5.0 \pm 1.2 \\
7.0 \pm 1.2 \\
2.6 \pm 1.2 \\
4.8 \pm 1.2 \\
6.9 \pm 1.2 \\
5.0 \pm 1.2\end{array}$ & $\begin{array}{r}1200 \pm 120 \\
880 \pm 90 \\
970 \pm 100 \\
450 \pm 50 \\
750 \pm 80 \\
740 \pm 70 \\
1100 \pm 110\end{array}$ & $\begin{array}{l}88 \pm 8 \\
56 \pm 5\end{array}$ & $\begin{array}{l}14 \pm 1 \\
23 \pm 2\end{array}$ \\
\hline $\begin{array}{l}1 \mathrm{NW} \\
2 \mathrm{NW} \\
3 \mathrm{NW} \\
4 \mathrm{NW} \\
5 \mathrm{NW} \\
6 \mathrm{NW}\end{array}$ & $\begin{array}{r}380 \pm 70 \\
270 \pm 50 \\
130 \pm 30 \\
170 \pm 30 \\
160 \pm 30 \\
80 \pm 20\end{array}$ & & & $\begin{array}{l}38 \pm 8 \\
170 \pm 33 \\
26 \pm 6 \\
26 \pm 6 \\
31 \pm 7 \\
13 \pm 3\end{array}$ & i & & $\begin{array}{r}240 \\
140 \\
1200 \\
290 \\
960 \\
380\end{array}$ & $\begin{array}{l} \pm 20 \\
\pm 10 \\
\pm 120 \\
\pm 30 \\
\pm 100 \\
\pm 40\end{array}$ & & $\begin{array}{r}2100 \pm 210 \\
910 \pm 90 \\
1900 \pm 190 \\
1300 \pm 130 \\
2000 \pm 200 \\
980 \pm 100\end{array}$ & $\begin{array}{l}3.5 \pm 1.2 \\
<1.2 \\
8.1 \pm 1.2 \\
4.3 \pm 1.2 \\
6.3 \pm 1.2 \\
2.8 \pm 1.2\end{array}$ & $\begin{array}{l}810 \pm 80 \\
880 \pm 90 \\
280 \pm 30 \\
120 \pm 10 \\
170 \pm 20 \\
850 \pm 90\end{array}$ & & \\
\hline
\end{tabular}

5 ansufficient sample for analysis where no data are reported. Uncertainties represent analytical uncertainties. 
TABLE A-III. Juniper: Elemental Concentrations ${ }^{\mathrm{a}}$

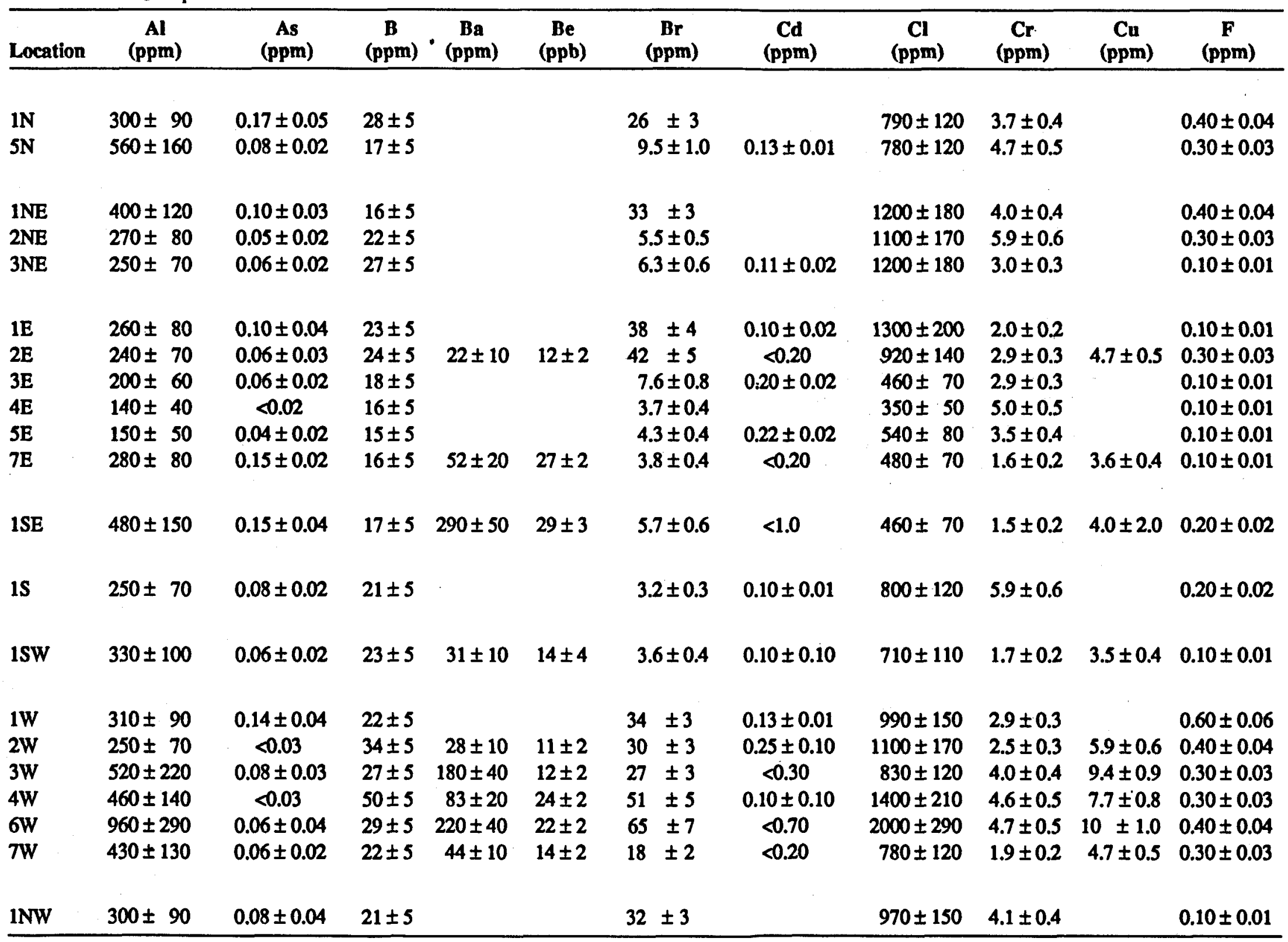




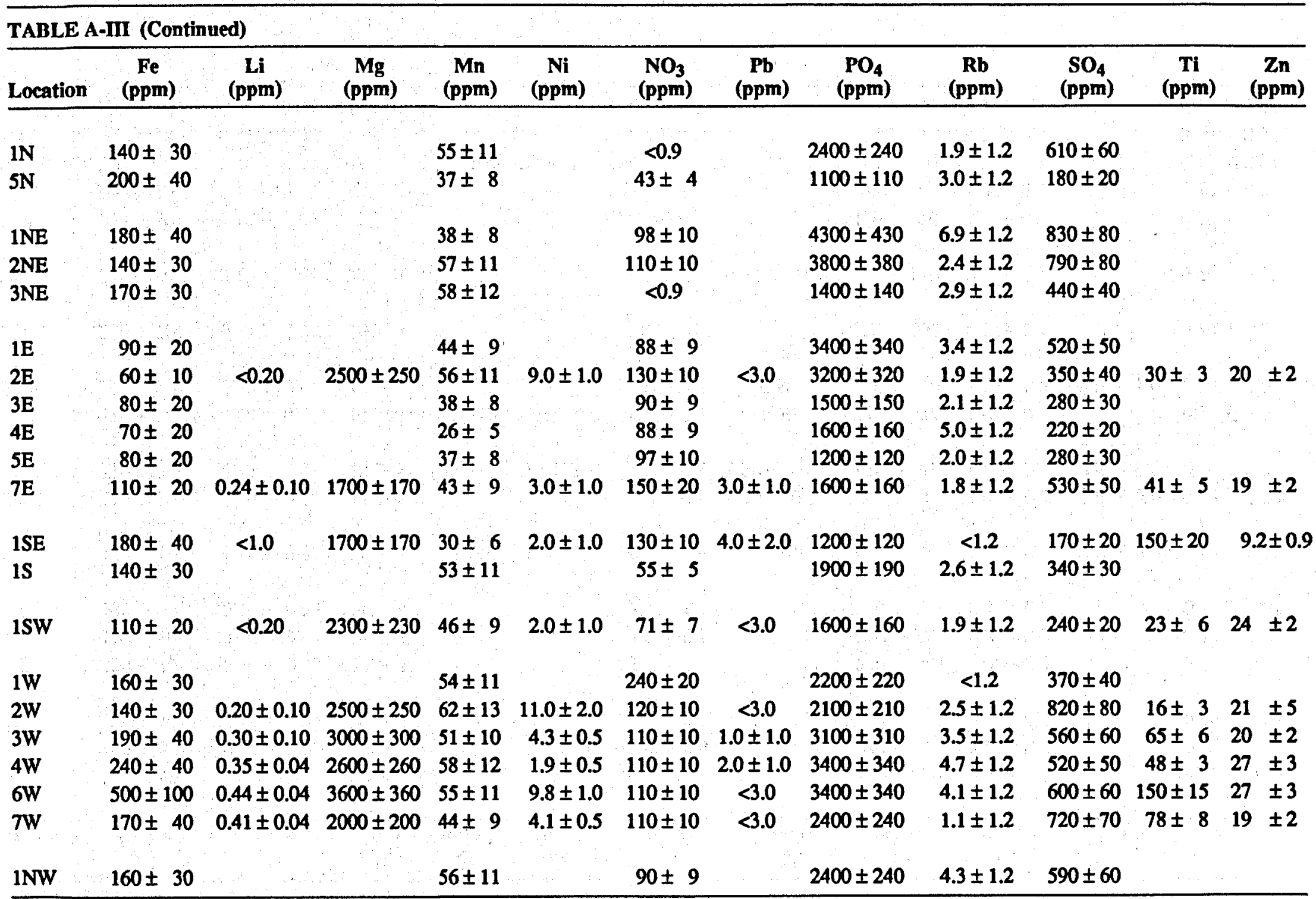

ansufficient sample for analysis where no data are reported. Uncertainties represent analytical uncertainties. 


\begin{tabular}{|c|c|c|c|c|c|c|c|c|c|c|c|c|}
\hline Location & $\begin{array}{c}\mathbf{A l} \\
(\mathbf{p p m})\end{array}$ & $\begin{array}{c}\text { As } \\
\text { (ppm) }\end{array}$ & $\begin{array}{c}\text { B } \\
(\mathbf{p p m})\end{array}$ & $\underset{(\mathrm{ppm})}{\mathrm{Ba}}$ & $\begin{array}{c}\text { Be } \\
(\mathbf{p p b})\end{array}$ & & $\begin{array}{l}\text { Br } \\
\text { opm) }\end{array}$ & $\underset{(\mathrm{ppm})}{\mathrm{Cd}}$ & $\begin{array}{c}\mathrm{Cl} \\
(\mathrm{ppm})\end{array}$ & $\begin{array}{c}\mathrm{Cr} \\
\text { (ppm) }\end{array}$ & $\underset{(\mathrm{ppm})}{\mathrm{Cu}}$ & $\begin{array}{c}F \\
(\mathrm{ppm})\end{array}$ \\
\hline $2 \mathrm{~N}$ & $140 \pm 40$ & $<0.03$ & $18 \pm 5$ & & & 19 & \pm 2 & & $320 \pm 50$ & $2.9 \pm 0.3$ & & $0.40 \pm 0.04$ \\
\hline $3 \mathbf{N}$ & $190 \pm 60$ & $0.09 \pm 0.03$ & $14 \pm 5$ & & & 7.9 & \pm 0.8 & & $230 \pm 30$ & $3.6 \pm 0.4$ & & $0.30 \pm 0.03$ \\
\hline $4 N$ & $130 \pm 40$ & $0.18 \pm 0.04$ & $22 \pm 5$ & & & 5.0 & \pm 0.5 & $0.10 \pm 0.01$ & $420 \pm 60$ & $3.1 \pm 0.3$ & & $0.30 \pm 0.03$ \\
\hline 4NE & $220 \pm 70$ & $0.15 \pm 0.03$ & $22 \pm 5$ & & & 15 & \pm 2 & & $280 \pm 40$ & $4.2 \pm 0.4$ & & $0.10 \pm 0.01$ \\
\hline 5NE & $140 \pm 40$ & $0.10 \pm 0.02$ & $21 \pm 5$ & & & 5.1 & \pm 0.5 & & $470 \pm 70$ & $2.8 \pm 0.3$ & & $0.10 \pm 0.01$ \\
\hline $6 \mathrm{NE}$ & $180 \pm 60$ & $0.11 \pm 0.03$ & $11 \pm 5$ & & & 9.9 & \pm 1 & & $330 \pm 50$ & $2.3 \pm 0.2$ & & $0.10 \pm 0.01$ \\
\hline $6 \mathrm{E}$ & $140 \pm 40$ & $0.15 \pm 0.02$ & $25 \pm 5$ & $27 \pm 10$ & $18 \pm 2$ & & \pm 0.6 & $<0.30$ & $400 \pm 60$ & $1.4 \pm 0.1$ & $2.2 \pm 0.2$ & $0.10 \pm 0.01$ \\
\hline 2SE & $250 \pm 70$ & $0.20 \pm 0.03$ & $17 \pm 5$ & & & & $1 \pm 0.5$ & $0.10 \pm 0.02$ & $260 \pm 40$ & $1.9 \pm 0.2$ & $7.0 \pm 3.0$ & $0.20 \pm 0.02$ \\
\hline 3SE & $310 \pm 90$ & $0.17 \pm 0.03$ & $28 \pm 5$ & $28 \pm 10$ & $13 \pm 2$ & & \pm 0.8 & $0.20 \pm 0.01$ & $370 \pm 60$ & $3.6 \pm 0.4$ & $2.5 \pm 0.3$ & $0.10 \pm 0.01$ \\
\hline $2 S$ & $230 \pm 70$ & $0.08 \pm 0.02$ & $19 \pm 5$ & $17 \pm 10$ & $8 \pm 2$ & & \pm 0.2 & $<0.20$ & $440 \pm 70$ & $1.0 \pm 0.1$ & & $0.10 \pm 0.01$ \\
\hline $3 S$ & $290 \pm 90$ & $0.30 \pm 0.04$ & $18 \pm 5$ & & & & \pm 0.7 & $<0.50$ & $230 \pm 40$ & $1.8 \pm 0.2$ & $4.0 \pm 1.0$ & $0.20 \pm 0.02$ \\
\hline 3SW & $220 \pm 70$ & $0.21 \pm 0.03$ & $46 \pm 5$ & & & & \pm 0.9 & & $4000 \pm 600$ & $4.1 \pm 0.4$ & & $0.20 \pm 0.02$ \\
\hline $5 W$ & $280 \pm 80$ & $0.19 \pm 0.04$ & $23 \pm 5$ & & & 55 & \pm 6 & $0.05 \pm 0.01$ & $710 \pm 110$ & $2.1 \pm 0.2$ & & $0.70 \pm 0.07$ \\
\hline $2 \mathrm{NW}$ & $100 \pm 30$ & $0.18 \pm 0.05$ & $17 \pm 5$ & & & 41 & \pm 4 & & $460 \pm 70$ & $6.8 \pm 0.7$ & & $0.10 \pm 0.01$ \\
\hline $3 N W$ & $290 \pm 90$ & $0.15 \pm 0.03$ & $17 \pm 5$ & & & & \pm 1 & & $390 \pm 60$ & $5.2 \pm 0.5$ & & $0.10 \pm 0.01$ \\
\hline $4 N W$ & $200 \pm 60$ & $0.14 \pm 0.04$ & $23 \pm 5$ & $<100$ & $24 \pm 4$ & 29 & \pm 3 & $<8.0$ & $450 \pm 70$ & $3.3 \pm 0.3$ & $<15$ & $0.10 \pm 0.01$ \\
\hline $5 N W$ & $230 \pm 70$ & $0.08 \pm 0.02$ & $25 \pm 5$ & & & & \pm 0.3 & & $250 \pm 40$ & $4.8 \pm 0.5$ & & $0.20 \pm 0.02$ \\
\hline $6 \mathrm{NW}$ & $120 \pm 40$ & $0.09 \pm 0.02$ & $23 \pm 5$ & & & & \pm 0.4 & & $330 \pm 50$ & $2.7 \pm 0.3$ & & $0.10 \pm 0.01$ \\
\hline
\end{tabular}




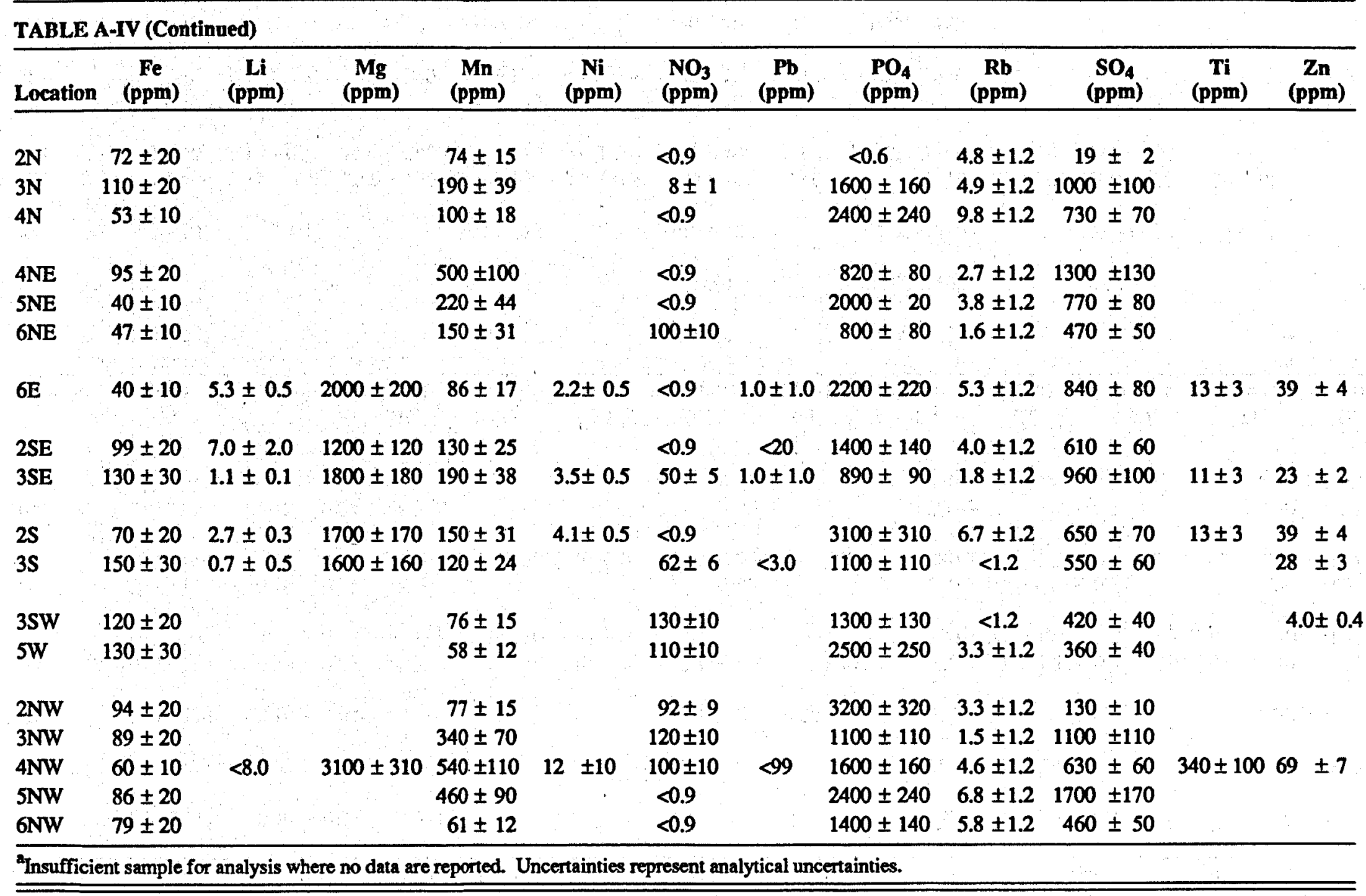


TABLE A-V. Oak: Elemental Concentrations ${ }^{2}$

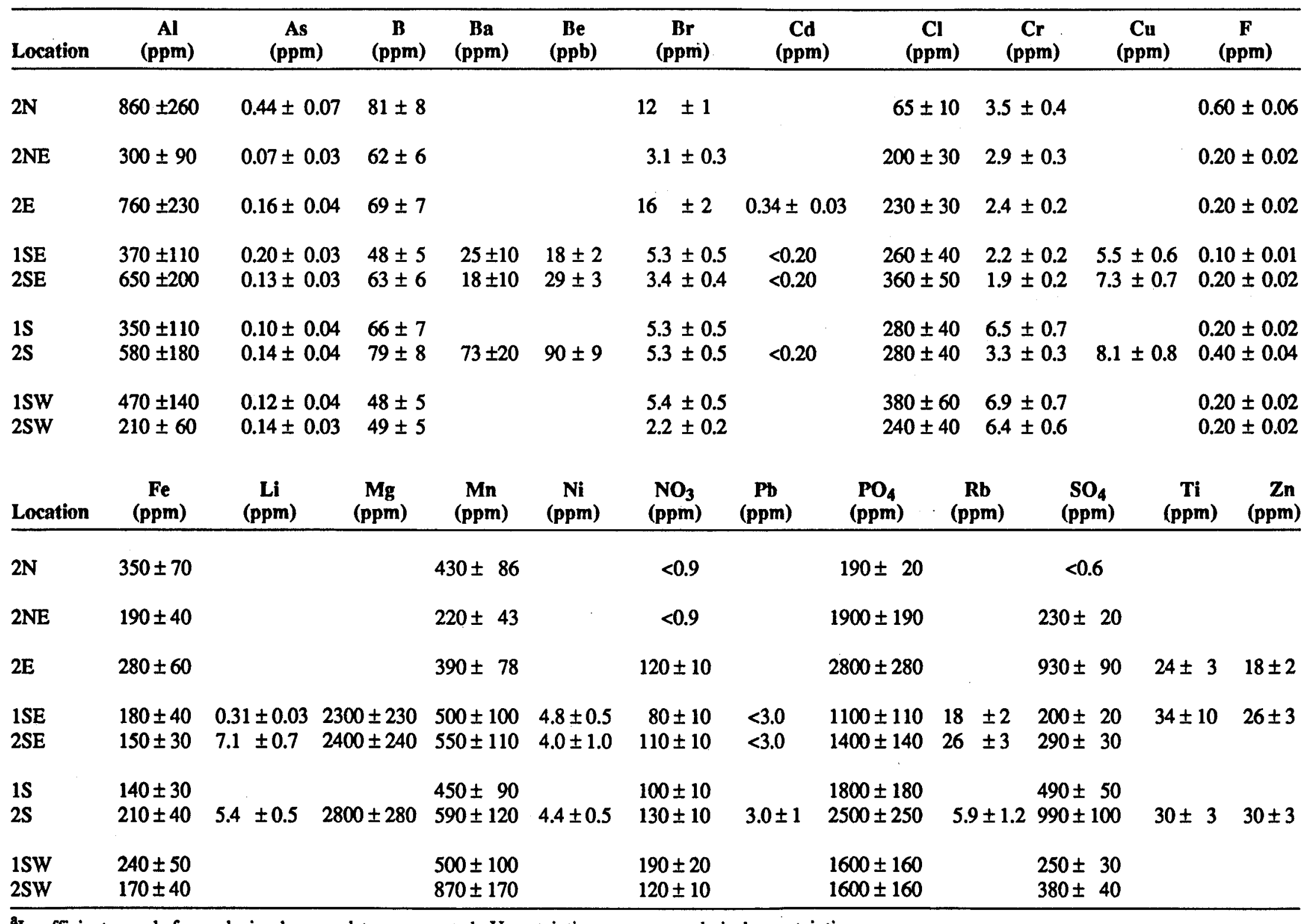

ansufficient sample for analysis where no data are reported. Uncertainties represent analytical uncertainties. 